\title{
Reproducibility and repeatability of assessment of myocardial light chain amyloidosis burden using ${ }^{18} \mathrm{~F}$-florbetapir PET/CT
}

\author{
Frank S. Prato, PhD, ${ }^{a, b}$ and Gerald Wisenberg, $M^{c}$ \\ a Departments of Medical Biophysics, Medical Imaging and Physics and Astronomy, Western \\ University, London, ON, Canada \\ b Lawson Imaging, Lawson Health Research Institute, London, ON, Canada \\ c MyHealth Centre, Arva, ON, Canada
}

Received Nov 18, 2019; accepted Nov 18, 2019

doi: 10.1007/s12350-019-01971-x

\section{See related article, pp. 2004-2010}

\section{INTRODUCTION}

In this edition of the JNC, Nodoushani et $\mathrm{al}^{1}$ have demonstrated the reproducibility and repeatability of ${ }^{18} \mathrm{~F}$-florbetapir PET imaging for the assessment of $\mathrm{AL}$ cardiac amyloidosis disease burden. To briefly review, Cardiac amyloidosis (CA) is a relatively rare condition ${ }^{2}$ in which amyloid protein precipitates throughout the myocardium. The disease is generally progressive and if not retarded or halted will invariably lead to both systolic and diastolic heart failure in rapid order. There are two major forms: (a) immunoglobulin light chain amyloid (AL) ${ }^{3}$ and (b) transthyretin amyloid (ATTR). ${ }^{4}$ The authors present the opinion that therapies for $\mathrm{AL}$ have been "limited" because imaging advances have lagged behind drug development.

However, the authors fail to provide supportive statements which verify the notion that, not only specific to the development of treatment for AL cardiac amyloidosis but also therapies for any disease, inadequate imaging tools contribute to the lack of successful treatment for that disease. To do so would require meeting three criteria: (1) the imaging modality accurately measures disease burden when compared against existing gold

\footnotetext{
Reprint requests: Frank S. Prato, PhD, Lawson Imaging, Lawson Health Research Institute, London, ON, Canada; prato@lawsonimaging.ca J Nucl Cardiol 2021;28:2011-3.

$1071-3581 / \$ 34.00$

Copyright (C) 2019 American Society of Nuclear Cardiology.
}

standard measurements; (2) these non-invasive measurements of disease burden (surrogate endpoints) accurately correlate with hard clinical endpoints-in the case of cardiac amyloid, this would include mortality rates, severity of left ventricular dysfunction, hospitalizations for heart failure and, most importantly; (3) that any treatment which uses imaging parameters as an endpoint, and claims benefit, is also associated with an improvement in the above noted clinical outcomes that correlate with the "improved" imaging parameters. As an example, in the recently presented ISCHEMIA trial, where imaging was used to identify those patients to undergo revascularization, outcomes did not improve. ${ }^{5}$

Imaging will undoubtedly play an ever increasing role in the assessment of patients with suspected cardiac amyloid, particularly as the index of suspicion for the presence of this entity has increased markedly over the past few years, ${ }^{2}$ as the diagnosis of the presence of diastolic heart failure has also similarly increased. Accurate early diagnosis and tracking of disease progression may play a role in improving patient outcomes while therapies are designed and assessed.

As AL and ATTR appear to have somewhat different clinical outcomes and response to therapy, ${ }^{4,6}$ there is some importance to being able to differentiate these two entities, if possible, with non-invasive imaging. With SPECT $/{ }^{99 \mathrm{~m}} \mathrm{Tc}$ Pyrophosphate, Ultrasound, and MRI, differentiation between AL and ATTR is difficult. Hence, the use of PET $/{ }^{18} \mathrm{~F}$-florbetapir may be an important screening tool to (a) initially make the diagnosis, (b) determine which subtype is present, (c) determine disease burden, and (d) aid in identifying patients for inclusion in clinical trials. Note that it has been shown that ${ }^{18} \mathrm{~F}$ florbetapir binds specifically to both AL and ATTR although a 50\% higher affinity for AL was shown. ${ }^{7}$

Early and accurate detection of amyloid may play an important role if it can be demonstrated that 
concurrent early introduction of effective therapies will ultimately alter patient outcomes. ${ }^{6}$ Hence, the reproducibility and repeatability of ${ }^{18} \mathrm{~F}$-florbetapir presented by the Brigham and Women's hospital group is very impressive, and given the superior resolution and sensitivity of PET over SPECT for cardiac, even with the new CZT pinhole cameras, ${ }^{8,9}$ it is unlikely that SPECT can be competitive to accomplish the goal of early disease detection. The intra- and inter-observer linear regressions that were demonstrated by the authors are outstanding especially when one considers that the readers were inexperienced, the Bland-Altman plot showed no offset and the Carimas software is freely available. Hence, provided quantitation of the amount of immunoglobin light chain amyloid protein is the correct metric to measure, ${ }^{18}$ F-florbetapir PET should be considered in future clinical trials of AL both to identify participants and to quantitate response.

It may be informative to the application of ${ }^{18} \mathrm{~F}$ florbetapir to $\mathrm{CA}$ by reflection on the successes and failure of the use of ${ }^{18} \mathrm{~F}$-florbetapir to quantitate amyloid in the brain as an endpoint in the many Alzheimer's Disease (AD) clinical trials. ${ }^{10}$ Even though ${ }^{18}$ F-florbetapir has demonstrated success of antibody therapy in removing amyloid, the important endpoint of impact on cognition did not, in most trials, track with changes in the ${ }^{18} \mathrm{~F}$-florbetapir results. Hence, some have argued that brain amyloid is not the appropriate imaging target, ${ }^{11,12}$ if in fact an appropriate imaging target exists. Intuitively, if amyloid deposition rates could be reduced or stopped, prior to significant permanent damage, favorable outcomes should ensue. This would argue for early diagnosis and early intervention before heart function is irreversibly compromised, and poor clinical outcomes are inevitable. Hence, there could be a significant value in ${ }^{18}$ F-florbetapir PET which in Nodoushani's preliminary study ${ }^{1}$ has shown outstanding reproducibility and repeatability, although we do not, as yet, have the evidence to support the belief that early diagnosis alone will, by itself, lead to better patient outcomes.

\section{RECOMMENDATIONS}

Although the results of Nodoushani et $\mathrm{al}^{1}$ are very encouraging, there is more that needs to be done:

(a) The inter-observer reproducibility and the intraobserver repeatability are based on reanalysis of the same data. As pointed out in this study, estimates based on repeat data acquisition on the same patient including a second injection of ${ }^{18} \mathrm{~F}$-florbetapir are needed. However, this will be a challenge given that: (i) most AL patients may be too sick to return for two scans on different days, (ii) it will be difficult to fit a second study between therapies, and (iii) normal volunteers cannot be used as diseasefree hearts do not take up the tracer.

(b) A similar study in patients with transthyretin amyloid (ATTR) should be undertaken, as, although this is a different disease, it makes up a significant CA burden ${ }^{2}$ and treatment is progressing, particularly if the disease is detected at an early stage. ${ }^{6}$ Note that for ATTR, Maurer et $\mathrm{al}^{4}$ acknowledge that PET with $11 \mathrm{C}$-PIB or ${ }^{18} \mathrm{~F}$-florbetapir or ${ }^{18} \mathrm{~F}$-florbetaben is more sensitive compared to SPECT, CMR, and Echocardiography.

(c) The relationship between ${ }^{18} \mathrm{~F}$-florbetapir uptake and clinical markers of cardiac function should be better determined, including biomarkers such as BNP levels. It is expected that significant uptake of ${ }^{18} \mathrm{~F}$ florbetapir will be detectable before functional changes are detected by US and MRI. Also, it will be important to investigate if ${ }^{18} \mathrm{~F}$-florbetapir uptake precedes changes in scar formation determined by delayed enhancement MRI which is a hallmark of permanent damage. ${ }^{13}$

(d) Initial diagnosis by ${ }^{18} \mathrm{~F}$-florbetapir might eliminate the need for endomyocardial biopsy and reduce the time to correct diagnosis which was estimated by Lousada et $\mathrm{al}^{14}$ to be greater than 6 months in $63 \%$ of cases. The practicality of using ${ }^{18} \mathrm{~F}$-florbetapir for diagnosis and avoidance of biopsy needs to be investigated. Given that ${ }^{18} \mathrm{~F}$-florbetapir has been widely available for dementia studies, accessibility should be acceptable. Of course, a major issue is cost. At current rates of about $\$ 2,000$ per dose (US) it is not competitive to the cost of SPECT. Significant reduction in cost might occur with the development of therapies that would be effective for the treatment of both brain and cardiac amyloid deposition.

The article by Noudashani ${ }^{1}$ is an important step in the development of an accurate image marker for cardiac amyloidosis which appears to be an important player in the development of heart failure in the elderly, in particular. ${ }^{2}$ However, we must also be careful to understand that in addition to focusing on improving the diagnostic accuracy of our imaging tools for this disease, we must also be sure that the use of such imaging endpoints guides us to improved patient outcomes.

\section{Disclosures}

Frank S. Prato and Gerald Wisenberg have nothing to disclose. 


\section{References}

1. Nodoushani A, El-Sady M, Park M, Castilloveitia G, Falk R, Di Carli M, et al. Reproducibility and repeatability of assessment of myocardial light chain amyloidosis burden using 18F-florbetapir PET/CT. J Nucl Cardiol 2019.

2. Gilstrap L, Dominici F, Wang Y, El-Sady M, Singh A, Di Carli M, et al. Epidemiology of cardiac amyloidosis-associated heart failure hospitalizations among fee-for-service medicare beneficiaries in the United States. Circ Hear Fail 2019;12(6):e005407.

3. Merlini G, Dispenzieri A, Sanchorawala V, Schonland S, Palladini G, Hawkins P, et al. Systemic immunoglobulin light chain amyloidosis. Nat Rev Dis Prim 2018;4(1):38.

4. Maurer M, Bokhari S, Damy T, Dorbala S, Drachman B, Fontana $\mathrm{M}$, et al. Expert consensus recommendations for the suspicion and diagnosis of transthyretin cardiac amyloidosis. Circ Heart Fail 2019;13(9):e006075.

5. Hochman J. Results for the ischemia trials: to intervene or not to intervene. In: American Heart Association Scientific Sessions [Internet]. 2019. p. 1-2. https://www.abstractsonline.com/pp8/\#!/ 7891/presentation/35079

6. Zhang K, Stockeri-Goldstein K, Lenihan D. Emerging therapeutics for the treatment of light chain and transthyretin amyloidosis. JACC Basic Transl Sci 2019;4(3):438-48.

7. Park M, Padera R, Belanger A, Dubey S, Hwang D, Veeranna V, et al. 18F-Florbetapir binds specifically to myocardial light chain and transthyretin amyloid deposits: autoradiography study. Circ Cardiovasc Imaging 2015;8(8):e002954.
8. Duvall W, Slomka P, Gerlach J, Sweeny J, Baber U, Croft L, et al. High-efficiency SPECT MPI: comparison of automated quantification, visual interpretation, and coronary angiography. J Nucl Cardiol 2013;20(5):763-73.

9. Alenazy A, Wells R, Ruddy T. New solid state cadmium-zinctelluride technology for cardiac single photon emission computed tomographic myocardial perfusion imaging. Expert Rev Med Devices 2017;14(3):213-22.

10. Chen W, Dilsizian V. Molecular imaging of amyloidosis: will the heart be the next target after the brain? Curr Cardiol Rep 2012;14(2):226-33.

11. Kametani F, Hasegawa M. Reconsideration of amyloid hypothesis and tau hypothesis in Alzheimer's disease. Front Neurosci 2018;12:25

12. Liu P, Xie Y, Meng X, Kang J. History and progress of hypotheses and clinical trials for Alzheimer's disease. Signal Transduct Target Ther 2019;4:29.

13. Kim RJ, Fieno DS, Parrish TB, Harris K, Chen EL, Simonetti O, et al. Relationship of MRI delayed contrast enhancement to irreversible injury, infarct age, and contractile function. Circulation 1999

14. Lousada I, Comenzo R, Landau H, Guthrie S, Merlini G. Light chain amyloidosis: patient experience survey from the amyloidosis research consortium. Adv Ther 2015;32:920-8.

Publisher's Note Springer Nature remains neutral with regard to jurisdictional claims in published maps and institutional affiliations. 